\title{
Opções de Compra: o Ajustamento ao Mercado Brasileiro de Dois Modelos de Precificação
}

\author{
Luiz Roque de Souza Vitiello Jr.
}

\section{RESUMO}

O presente estudo verificou, para o período de julho de 1994 a junho de 1997, o ajustamento ao mercado brasileiro de dois modelos de precificação de opções de compra: o de Black e Scholes (1973), que reconhece que os retornos dos preços da ação-objeto seguem um processo de difusão log-normal e o proposto por Cox e Ross (1976), que admite um processo de difusão com elasticidade da variância constante. Com a utilização de diferenças percentuais relativas e do teste t, observou-se que ambos os modelos subprecificaram opções fora do preço e no preço, enquanto, para opções dentro do preço, somente as mais próximas do vencimento foram subprecificadas. Verificou-se que o modelo de Black e Scholes (1973) ajustou-se melhor às opções fora do preço e no preço, enquanto o modelo da elasticidade da variância constante ajustou-se melhor às opções dentro do preço. Por fim, os resultados sugerem que, embora o modelo de Black e Scholes (1973) se ajuste ligeiramente melhor ao mercado brasileiro, de uma forma geral, não se pode afirmar que um modelo seja superior ao outro na precificação de opções de compra.

Palavras-chaves: opções de compra; Black e Scholes; elasticidade da variância constante.

\begin{abstract}
This study examined, from July 1994 to June 1997, the goodness of fitting to the Brazilian market of two call option pricing models: the Black and Scholes (1973), which assumes that the returns of the underlying stock prices follow a lognormal diffusion process, and the constant elasticity of variance, suggested by Cox and Ross (1976), which assumes that the returns of the underlying stock prices follow a constant elasticity of variance diffusion process. Through the use of relative percentage differences and the t-test, it was verified that both models underpriced options out of the money and in the money whereas for at the money options, only the closest to the expiration day were underpriced. It was also verified that the Black and Scholes (1973) model adjusted better to out of the money and at the money options whereas the constant elasticity of variance model fitted better to in the money options. Finally, the results suggest that, although the Black and Scholes (1973) model performs slightly better than the other, it cannot be said that one model fits better to the market than the other in the pricing of Brazilian call options.
\end{abstract}

Key words: call options; Black and Scholes; constant elasticity of variance. 


\section{INTRODUÇĀO}

Os mercados derivativos propiciam aos agentes econômicos oportunidades para gerenciar e transferir riscos. Sendo um tipo de ativo derivativo, uma opção proporciona ao proprietário, mediante um custo, chamado prêmio, o direito, e não a obrigação, de comprar ou vender uma determinada quantidade do ativo-objeto a um preço pré-definido.

Para que os negociadores de ativos derivativos possam atingir os objetivos que se propõem - proteção, especulação ou arbitragem, conforme Hull (1993) - uma correta valoração do prêmio pago demonstra ser relevante, porque, do contrário, pode-se estar pagando um preço não justo pela opção, aumentando ou reduzindo o custo da proteção ou da alavancagem de um portfólio, bem como possibilitando oportunidades de arbitragem.

Por serem ativos derivativos, opções sobre ações dependem do comportamento da ação-objeto para que possam ser precificadas. Partindo de premissas sobre os processos de formação dos preços da ação-objeto, diferentes modelos de precificação de ações foram desenvolvidos.

A posse de maior conhecimento do modelo de precificação de opções, que expresse mais claramente o comportamento das opções negociadas no mercado brasileiro, pode auxiliar os agentes econômicos na tomada de decisão e contribuir para a eficiência do mercado, evitando a obtenção de retornos anormais por parte dos agentes econômicos. Na tentativa de colaborar para a solução desse problema, utilizam-se, neste estudo, dois modelos de precificação.

O primeiro modelo, desenvolvido por Black e Scholes (1973), admite que o preço das ações segue um processo de difusão log-normal, com variância constante dos retornos ao longo do tempo. Apesar de exigir a estimativa de apenas um único parâmetro (a volatilidade dos retornos da ação), foram observados vieses sistemáticos na utilização do modelo, com relação ao preço de exercício, ao prazo para o vencimento da opção e, principalmente, à volatilidade da ação-objeto.

O segundo modelo, proposto por Cox e Ross (1976), conhecido como modelo de precificação com a elasticidade da variância constante (constant elasticity of variance-CEV), procura explicar os vieses do modelo de Black e Scholes (1973), flexibilizando o pressuposto da variância constante ao longo do tempo, presumindo que os retornos do ativo-objeto são heterocedásticos. De acordo com o modelo, 
a taxa de variância da ação não é constante ao longo do tempo, sendo função decrescente do preço da ação.

Com o presente estudo, procura-se verificar se os preços calculados pelos modelos se ajustam àqueles praticados pelo mercado brasileiro. Em particular, dados os vieses relatados do modelo de Black e Scholes (1973), verificar se o modelo proposto por Cox e Ross (1976) consegue explicar, de forma mais precisa, o comportamento dos preços das opções de compra negociadas na Bolsa de Valores do Estado de São Paulo (Bovespa), para os exercícios de outubro de 1994 a junho de 1997, inclusive. Ressalta-se que, embora os preços de opções de venda também possam ser calculados, estas apresentam baixíssima liqüidez no mercado brasileiro, motivo pelo qual o estudo se utilizou apenas de opções de compra.

\section{Precificação de Opçóes}

Opções, sendo ativos derivativos ${ }^{(1)}$, dependem do preço da ação-objeto para serem precificadas. De acordo com o processo que se adota para a formação dos retornos dos preços da ação-objeto, diferentes resultados podem ser encontrados na valoração de opções. Pretende-se apresentar, nesta etapa, o referencial teórico, o desenvolvimento matemático e as particularidades dos modelos estudados: o de Black e Scholes (1973), doravante BS, que aceita que os preços da ação-objeto seguem um processo de difusão ${ }^{(2)} \log$-normal e o proposto por Cox e Ross (1976), que admite um processo de difusão com elasticidade da variância constante.

\section{O Modelo de Black e Scholes}

Baseando-se em um modelo geral de equilíbrio, Black e Scholes (1973) desenvolveram a primeira fórmula explícita para precificar opções, utilizando-se de pressupostos restritivos para comportamento dos ativos.

Para derivar a fórmula de precificação de opções, Black e Scholes (1973) criaram um portfólio, ajustado continuamente, composto por uma posição comprada em uma unidade da ação-objeto e uma vendida em $1 /(\partial \mathrm{c} / \partial \mathrm{S})$ unidades de opções, onde S é o preço da ação e c é o preço da opção. Neste caso, considera-se o portfólio como sendo livre de risco porque, em curto espaço de tempo, os dois ativos são afetados pela mesma fonte de incerteza. O portfólio, portanto, deve ter rendimento igual à taxa livre de risco, pois não depende do preço da ação mas somente do tempo e dos valores constantes conhecidos.

O movimento dos preços da opção ao longo do tempo, por sua vez, sendo fun- 
ção dos preços da ação-objeto, deve estar relacionado ao movimento dos preços desta. Se o movimento nos preços das ações segue um processo de Wiener generalizado (também conhecido como movimento browniano geométrico), o retorno da ação-objeto pode ser descrito como:

Equação 1

$$
\frac{\mathrm{dS}}{\mathrm{S}}=\mu \mathrm{dt}+\sigma \mathrm{dz}
$$

onde $\mu$ é a média instantânea, $\sigma$ é o desvio-padrão instantâneo (sendo $\sigma^{2}$ a variância instantânea), $\mathrm{z}$ o processo de Wiener e $\mathrm{t}=\mathrm{T}-\tau$, onde t é período de tempo para o vencimento da opção, $\mathrm{T}$ é a data de vencimento e $\tau$ é a data atual.

Considerando-se a formação do portfólio livre de risco, o processo descrito na Equação 1 e as restrições:

$$
\begin{aligned}
\mathrm{c} & =\mathrm{S}_{\mathrm{T}}-\mathrm{X}, & & \mathrm{S}_{\mathrm{T}} \geq \mathrm{X} \\
& =0, & & \mathrm{~S}_{\mathrm{T}}<\mathrm{X},
\end{aligned}
$$

onde X é preço de exercício, Black e Scholes (1973) estabeleceram a fórmula para precificação de opções de compra, dada por:

Equação 2

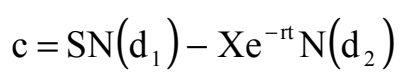

onde:

$$
\mathrm{d}_{1}=\frac{\ln (\mathrm{S} / \mathrm{X})+\left[\mathrm{r}+\left(\sigma^{2} / 2\right)\right] \mathrm{t}}{\sigma \sqrt{\mathrm{t}}} ; \quad \mathrm{d}_{2}=\mathrm{d}_{1}-\sigma \sqrt{\mathrm{t}} ; \quad \mathrm{r} \text { : taxa livre de risco }
$$

$\mathrm{N}(\mathrm{x})$ : probabilidade cumulativa de uma variável normal padronizada.

\section{O Modelo da Elasticidade da Variância Constante}

Cox e Ross (1976) e Cox (1996) demonstram a valoração de opções por meio da utilização de processo de difusão com elasticidade da variância constante, como forma de modelar a heterocedasticidade dos retornos das ações: ao con- 
trário dos pressupostos do modelo $\mathrm{BS}$, o modelo $\mathrm{CEV}$ considera que a variância da taxa de retorno das ações depende das mudanças dos preços dessas nos períodos anteriores.

Matematicamente, o processo de difusão com elasticidade da variância constante, para $0 \leq \theta<2$ e $\delta>0$, pode ser descrito da seguinte forma:

Equação 3

$$
\mathrm{dS}=\mu \mathrm{Sdt}+\delta \mathrm{S}^{\theta / 2} \mathrm{dz}
$$

onde $\theta$ é a elasticidade da variância instantânea com relação ao preço da ação e o quadrado do último termo é a variância instantânea do preço da ação, dada por $\sigma^{2}(\mathrm{~S}, \mathrm{t})=\delta^{2} \mathrm{~S}^{\theta}$.

A variância instantânea dos retornos, para este processo, é dada por $\delta^{2} S^{(\theta-2)}$, sendo uma função decrescente do preço da ação para $\theta<2$. Quando $\theta=2$ (ou seja, a elasticidade é zero), a variância instantânea passa a ser constante e o processo torna-se de difusão log-normal (Macbeth e Merville, 1980). O modelo BS, portanto, é um caso especial do modelo CEV.

Sendo a probabilidade de $\mathrm{S}_{\mathrm{T}}=0$ dada por $G(1 /(2-\theta) \omega$, onde $G(m, v)=[\Gamma(m)]^{-1} \int_{v} e^{-u} u^{m-1} d u$ é a função distribuição gama complementar, percebe-se que, à medida que $\theta$ se aproxima de dois, essa probabilidade aproxima-se de zero. Por fim, considerando-se que $\mathrm{c}=\max \left(\mathrm{S}_{\mathrm{T}}-\mathrm{X}, 0\right)$ e descontando-se ao instante $\tau$, a fórmula para precificação de uma opção de compra pode ser representada por (Cox e Ross, 1976):

Equação 4

$$
\mathrm{c}=\mathrm{S} \sum_{\mathrm{n}=0}^{\infty} \frac{\mathrm{e}^{-\omega} \omega^{\mathrm{n}} \mathrm{G}\left(\mathrm{n}+1+\frac{1}{2-\theta}, \mathrm{kX}^{2-\theta}\right)}{\Gamma(\mathrm{n}+1)}-\mathrm{Xe}^{-\mathrm{rt}} \sum_{\mathrm{n}=0}^{\infty} \frac{\mathrm{e}^{-\omega} \omega^{\mathrm{n}+\frac{1}{2-\theta}} \mathrm{G}\left(\mathrm{n}+1, \mathrm{kX} \mathrm{X}^{2-\theta}\right)}{\Gamma\left(\mathrm{n}+1+\frac{1}{2-\theta}\right)}
$$

onde:

$$
\mathrm{k}=\frac{2 \mathrm{r}}{\delta^{2}(2-\theta)\left[\mathrm{e}^{\mathrm{rt}(2-\theta)}-1\right]} ; \quad \omega=\mathrm{kS}_{\tau}^{2-\theta} \mathrm{e}^{\mathrm{rt}(2-\theta)} ; \quad \mathrm{z}=\mathrm{kS}_{\mathrm{T}}^{2-\theta}
$$




\section{Evidéncias Empíricas}

Estudos têm demonstrado que o modelo BS apresenta vieses sistemáticos em relação ao preço de exercício, ao prazo para o vencimento da opção e, principalmente, à volatilidade da ação-objeto (único parâmetro que necessita ser estimado). Outros têm demonstrado que o modelo CEV tem a capacidade de precificar opções de forma mais precisa que o modelo BS.

Procurando testar empiricamente o próprio modelo, Black e Scholes (1972) verificaram que a utilização da variância estimada por meio de dados passados pode causar a superavaliação das opções sobre as ações muito voláteis e a subavaliação das opções sobre as ações pouco voláteis. De acordo com os autores, estimando-se a variância da ação por meio de dados históricos, que compreendam somente o período de vida da opção, obtém-se uma redução do viés.

Posteriormente, Black (1975), analisando o comportamento do modelo BS, verificou que opções que estão dentro (fora) do preço tendem a ser superavaliadas (subavaliadas) pelo modelo, enquanto opções com menos de três meses para o vencimento tendem a ser subavaliadas ${ }^{(3)}$. O autor também observou que "a volatilidade de uma ação pode variar ao longo do tempo e que outros fatores, além da volatilidade histórica, podem ser úteis no prognóstico" (Black, 1975, p. 36). Por fim, sugere que, se a volatilidade histórica não é condizente com a realidade, deve ser porque o mercado sabe algo sobre a volatilidade futura que nós não sabemos; portanto a utilização da volatilidade implícita deveria ser considerada na estimativa de volatilidade futura da ação.

Macbeth e Merville (1979) compararam os preços de opções de compra, lançadas sobre seis ações negociadas na Chicago Board of Trade Options Exchange, com os preços calculados pela fórmula de BS, ajustados para o pagamento de dividendos, para o período de 31 de dezembro de 1975 a 31 de dezembro de 1976. Os autores verificaram que o modelo BS, em média, subavaliava opções dentro do preço e superavaliava opções fora do preço, enquanto opções com menos de três meses para o vencimento eram superavaliadas. Os autores reconhecem que os resultados obtidos no estudo são opostos aos alcançados por Black (1975), mas acreditam que a diferença dos resultados pode ser justificada, pelo menos em parte, pela não estacionariedade da taxa de variância do processo estocástico gerador dos preços da ação.

Utilizando-se dos preços de fechamento para todas as opções de compra sobre seis ações, negociadas na Chicago Board of Trade Options Exchange, no período de 31 de dezembro de 1975 a 31 de dezembro de 1976, Macbeth e Merville (1980) 
compararam os preços teóricos dos modelos BS e CEV (ajustados ao pagamento de dividendos) com os preços de opções praticados pelo mercado. Os autores verificaram que o valor da variável $\theta$ (do modelo CEV) é geralmente inferior a dois e que o modelo CEV demonstrou ser superior ao modelo BS na precificação das opções de compra da amostra. Com base nos resultados obtidos, os autores concluíram que os preços das ações seguem um processo de difusão com elasticidade da variância constante com parâmetro $\theta$ geralmente inferior a dois.

Calculando a variância implicitamente ${ }^{(4)}$, Barreto e Baidya (1987) testaram o modelo BS na precificação de opções de compra da Vale do Rio Doce, negociadas na Bolsa de Valores do Rio de Janeiro, com exercícios durante o ano de 1986. Os autores concluíram que, em relação ao preço teórico fornecido pela fórmula BS, o mercado superavalia (subavalia) opções fora do preço (dentro do preço) sistematicamente. Os resultados estão de acordo com os obtidos por Black (1975) e contrariam os obtidos por Macbeth e Merville (1979).

Outro estudo, realizado por Becker e Lemgruber (1987), verificou a capacidade de ajustamento do modelo BS aos preços das opções de compra lançadas sobre três ações negociadas na Bolsa de Valores do Estado de São Paulo, para o ano de 1986. Para uma amostra composta de 46 séries de opções, os autores constataram que o modelo subavaliou o preço das opções para $86 \%$ das observações. A diferença média entre o preço calculado pelo modelo e o praticado pelo mercado era de $38 \%$, sendo considerada significativamente diferente de zero (teste t). Becker e Lemgruber (1987) demonstraram, no entanto, que grande parte desta diferença residia na assimetria da distribuição dos erros e, portanto, esses não poderiam ser modelados por uma distribuição simétrica.

Os preços das opções de compra sobre moedas foram comparados, por Tucker, Peterson e Scott (1988), aos preços calculados pelos modelos BS e CEV (ambos adaptados ao mercado de moedas). Calculando a variância das opções implicitamente, os autores verificaram que o modelo $\mathrm{CEV}$ proporcionou resultados mais precisos do que o modelo BS.

Testando empiricamente o modelo BS, ajustado à precificação de warrants e à distribuição de dividendos, Lauterbach e Schultz (1990) utilizaram-se de uma amostra composta por 39 warrants, negociadas no período de janeiro de 1971 a dezembro de 1980, totalizando 25.171 observações diárias. Os autores verificaram a existência de uma relação inversa entre o desvio-padrão e o preço da ação e concluíram, ao comparar os modelos BS e CEV, $\operatorname{com} \theta$ fixado arbitrariamente em 1, que o pressuposto da elasticidade constante da variância é a maior deficiência do modelo BS e que, portanto, o modelo CEV supera aquele na precificação de warrants. 
Swidler e Diltz (1992) procuraram verificar a consistência do modelo BS, ajustado para determinada taxa de crescimento de dividendos, $\mathrm{g}$, em relação às opções negociadas na Chicago Board of Trade Options Exchange. Os autores verificaram que as taxas baseadas nos preços de oferta de venda são maiores do que as de compra, evidenciando que as taxas implícitas de crescimento de dividendos não são condizentes com o pressuposto da volatilidade constante em relação ao tempo, do modelo BS. Swidler e Diltz (1992) concluem que os resultados obtidos sustentam a utilização do modelo CEV.

Hauser e Lauterbach (1997) compararam o desempenho relativo de cinco fórmulas de precificação de warrants: BS; BS ajustado para diluição; CEV ajustado $\operatorname{com} \theta=1 ;$ CEV ajustado com $\theta$ estimado e o modelo de Longstaff (apud Hauser e Lauterbach, 1997). Foi utilizada a amostra da pesquisa de Lauterbach e Schultz (1990), composta por 29 warrants e mais de 23 mil observações. Os autores concluíram que, entre os modelos estudados, o modelo CEV apresentou os melhores resultados. Hauser e Lauterbach (1997) atentam para o fato da complexidade dos cálculos exigidos pelo modelo CEV, e acreditam que ele é apenas um ponto intermediário em uma linha de progresso para melhores modelos de precificação.

\section{Metodologia}

Uma vez concluída a revisão dos referenciais teóricos e das pesquisas empíricas realizadas, parte-se para a descrição da amostra e o detalhamento da metodologia empregada.

\section{Amostra}

Para a composição da amostra, foram utilizados os preços de fechamento das ações-objetos e os preços de fechamento e de exercício das opções de compra lançadas sobre essas ações-objetos, negociadas na Bolsa de Valores do Estado de São Paulo (Bovespa), no período compreendido entre julho de 1994 e junho de 1997, inclusive.

Os preços das ações e das opções foram coletados a partir do quinto dia útil antes do vencimento $\mathrm{T}_{-5}$, em intervalos de cinco dias úteis, até o sexagésimo dia útil antes do vencimento $\mathrm{T}_{-60}:$ a partir do dia de vencimento $\mathrm{T}$, os preços foram coletados nos instantes $t_{5}, t_{10}, t_{15}, \ldots, t_{50}, t_{55}, t_{60}$. Os preços das ações, das opções e os de exercício foram coletados do jornal Gazeta Mercantil. 
Foram excluídas da amostra opções que não se ajustavam aos princípios de arbitragem do modelo BS e opções com preços inferiores a $\mathrm{R} \$ 1,00^{(5)}$. Também foram excluídas da amostra as observações que, em determinado dia, tenham sido a única série negociada sobre a mesma ação. Com tais ajustes, a amostra totalizou 767 observações.

Para definir se uma ação está dentro do preço, no preço ou fora do preço, utilizou-se a seguinte relação:

Equação 5

$$
\mathrm{M}=\frac{\mathrm{S}}{\mathrm{Xe}}
$$

De forma semelhante à utilizada por Hauser e Lauterbach (1997), considera-se, arbitrariamente, uma opção dentro do preço, se o resultado da Equação 5 for maior que 1,05 , ou seja $M>1,05$. Se o resultado for menor ou igual a $0,95(M \leq$ $0,95)$, a opção está fora do preço. Para os demais casos $(0,95<\mathrm{M} \leq 1,05)$, a opção é considerada no preço.

Como taxa livre de risco foi utilizado o Certificado de Depósito Interbancário over de um dia, divulgado na forma de taxa over que, de acordo com Fortuna (1996, p. 77), "são os títulos de emissão das instituições financeiras que lastreiam as operações do mercado interbancário".

No Brasil, como as opções são protegidas contra o pagamento de dividendos, as fórmulas de precificação de opções podem ser aplicadas diretamente, não sendo necessário, portanto, qualquer tipo de ajuste ou correção. Embora as opções de compra negociadas no mercado brasileiro sejam americanas, Merton (1973) demonstra que nunca é ótimo exercer uma opção americana, que não paga dividendos, antes do vencimento; conforme Hull (1993), opções americanas são aquelas que podem ser exercidas a qualquer tempo antes e na data de vencimento.

\section{Estimativa do Parâmetro do Modelo de Black e Scholes}

Uma vez que o modelo BS exige a estimativa da volatilidade da ação-objeto, foi utilizado o desvio-padrão implícito do modelo. Como não é possível isolar a variável $\sigma$, na Equação 2, utilizou-se, inicialmente, um método de procura numérica que igualasse o preço fornecido pela fórmula ao preço praticado pelo mercado.

Tendo-se obtido o desvio-padrão implícito de cada uma das opções, empregouse a Equação 6 para a obtenção de um único desvio-padrão sobre cada ação, para 
cada dia de negociação; de acordo com Macbeth e Merville (1979), essa equação retorna o desvio-padrão implícito de uma opção de compra no preço.

Equação 6

$$
\sigma_{\mathrm{ijt}}=\sigma_{\mathrm{i} 0 \mathrm{t}}+\sigma_{\mathrm{i} 1 \mathrm{t}} \mathrm{m}_{\mathrm{ijt}}+\epsilon_{\mathrm{ijt}} \quad \mathrm{j}=1,2, \ldots, \mathrm{J}
$$

onde $\sigma_{\mathrm{ijt}}$ é o desvio-padrão implícito da opção j, sobre a ação i, no instante t, calculada pelo modelo BS, $\sigma_{\mathrm{i} 0 \mathrm{t}}, \sigma_{\mathrm{i} 1 \mathrm{t}} \mathrm{e} \in \in_{\mathrm{ijt}}$ são parâmetros da regressão e $\mathrm{m}_{\mathrm{ijt}}=\frac{\mathrm{S}_{\mathrm{it}}-\mathrm{X}_{\mathrm{ij}} \mathrm{e}^{-\mathrm{rt}}}{\mathrm{X}_{\mathrm{ij}} \mathrm{e}^{-\mathrm{r \tau}}}$, sendo $S_{i t}$ o preço da ação i no instante te $X_{\mathrm{ij}} \mathrm{e}^{\text {-rt }}$ é o valor presente, no instante t, do preço de exercício da opção j sobre a ação i.

Os valores anualizados, obtidos para o parâmetro $\sigma$, se encontraram entre o valor mínimo de $5 \%$ e máximo de $195 \%$, apresentando média e mediana de $32 \%$ e $38 \%$, respectivamente.

\section{Estimativa dos Parâmetros do Modelo CEV}

Enquanto o modelo BS exige a estimativa de apenas um parâmetro, $\sigma$, o modelo CEV exige a estimativa de dois: $\theta \mathrm{e} \delta$, ambos relacionados à volatilidade da açãoobjeto.

Para o modelo CEV, assim como o modelo BS, foi utilizado o desvio-padrão implícito da opção como estimativa da variância da ação; entretanto, visto que a Equação 4 possui duas variáveis incógnitas, $\theta$ e $\delta$, empregou-se a mesma metodologia adotada por Macbeth e Merville (1980), Tucker, Peterson e Scott (1988) e Lauterbach e Schultz (1990): utilizou-se a variância implícita do modelo BS como parâmetro de entrada para o modelo CEV.

Para a estimativa do parâmetro $\theta$, partindo-se da variância implícita calculada pelo modelo BS, para cada uma das opções de compra constantes da amostra, fez-se

Equação 7

$$
\delta^{2}=\frac{\sigma^{2}(\mathrm{~S}, \mathrm{t})}{\mathrm{S}^{(\theta-2)}}
$$

onde $\sigma^{2}(\mathrm{~S}, \mathrm{t})$ é a variância implícita do modelo BS. Substituiu-se a Equação 7 na Equação 4, obtendo-se, desta forma, uma equação e uma única incógnita, o próprio parâmetro $\theta$. Utilizou-se, então, um processo de procura numérica para achar 
um valor de $\theta$ que minimizasse o quadrado da diferença entre o preço teórico da opção (calculado de acordo com o modelo CEV) e o preço de mercado desta mesma opção. Embora a Equação 4 envolva duas séries numéricas, a convergência ocorre, em média, com 800 iterações.

Considerando-se que o valor obtido para $\theta$, a partir de uma opção de compra no preço, seja uma estimativa bastante razoável para o verdadeiro valor de $\theta$, e visto que uma opção no preço não é facilmente observável, aplicou-se a seguinte regressão para se obter uma estimativa de $\theta$ para uma opção no preço ${ }^{(6)}$ (Tucker, Peterson e Scott, 1988):

Equação 8

$$
\theta_{\mathrm{ijt}}=\theta_{\mathrm{i} 0 \mathrm{t}}+\theta_{\mathrm{i} 1 \mathrm{t}} \mathrm{m}_{\mathrm{ijt}}+\epsilon_{\mathrm{ijt}} \quad \mathrm{j}=1,2, \ldots, \mathrm{J}
$$

onde $\theta_{\mathrm{i} 0 \mathrm{t}}$ é o teta implícito da opção j, sobre a ação i, no instante t, calculada pela substituição da Equação 7 na Equação 4.

Dos valores obtidos para o parâmetro $\theta$, dado o intervalo $[0 ; 1,9)$, aproximadamente $52 \%$ encontraram-se no intervalo $(1,6 ; 1,8]$ e $72 \%$ no intervalo $(1,5 ; 1,9]$.

A constante $\delta$ foi estimada, para cada dia t, a partir da estimativa das variáveis $\hat{\sigma}_{\mathrm{i} 0 \mathrm{t}}$ (calculada de acordo com a Equação 6) e da variável $\theta$, resultando, em ambos os casos, em uma opção no preço.

\section{Equaçóes Utillizadas no Teste de Comparaçăo}

Definem-se a diferença percentual relativa dos preços teóricos das opções de compra em relação aos preços praticados pelo mercado e a diferença percentual relativa entre os preços calculados pelos modelos, respectivamente, como:

Equação 9

$$
\begin{aligned}
F_{(\text {mod elo }) \mathrm{ijt}} & =\frac{\left(\mathrm{P}_{(\text {mod elo }) \mathrm{ijt}}-\mathrm{P}_{(\text {mercado }) \mathrm{ijt}}\right)}{\mathrm{P}_{(\text {mercado }) \mathrm{ijt}}} \\
\mathrm{F}_{\text {mijt }} & =\frac{\left(\mathrm{P}_{(\mathrm{CEV}) \mathrm{ijt}}-\mathrm{P}_{(\mathrm{BS}) \mathrm{ijt}}\right)}{\mathrm{P}_{(\mathrm{BS}) \mathrm{jit}}}
\end{aligned}
$$

Equação 10 
onde i é o preço da ação, j é o preço da opção, t é o instante, $\mathrm{P}_{(\text {modelo)jit }}$ é o preço teórico da opção de compra calculado pelo modelo BS ou CEV, $\mathrm{P}_{(\text {mercado)jij }}$ é o preço de mercado da opção de compra, $\mathrm{P}_{(\mathrm{CEV}) \mathrm{jij}}$ é o preço teórico da opção de compra calculado conforme o modelo CEV e $\mathrm{P}_{(\mathrm{BS}) \mathrm{jij}}$ é o preço teórico da opção de compra calculado conforme o modelo BS. A média das diferenças percentuais relativas, para $\mathrm{F}_{(\text {modelo)jit }}$ e $\mathrm{F}_{\text {mijt }}$ é representada respectivamente por:

Equação 11

$$
\mathrm{R}_{(\text {mod elo) })}=\frac{1}{\mathrm{k}} \sum_{\mathrm{i}=1}^{\mathrm{k}} \frac{1}{\mathrm{n}} \sum_{\mathrm{j}=1}^{\mathrm{n}} \mathrm{F}_{(\text {mod elo }) \mathrm{jit}} \quad \mathrm{t}=5,10, \ldots, 55,60
$$

Equação 12

$$
\mathrm{R}_{\mathrm{mt}}=\frac{1}{\mathrm{k}} \sum_{\mathrm{i}=1}^{\mathrm{k}} \frac{1}{\mathrm{n}} \sum_{\mathrm{j}=1}^{\mathrm{n}} \mathrm{F}_{\mathrm{mijt}}
$$$$
\mathrm{t}=5,10, \ldots, 55,60
$$

onde k e $\mathrm{n}$ representam, respectivamente, o número de ações e opções para o dia t.

Essas equações colocam o teste da seguinte forma: se os modelos precificam as opções de forma semelhante, o resultado para as equações acima deverá aproximar-se de 0 .

\section{Resultados}

No teste realizado, aplicou-se a Equação 9 aos preços teóricos calculados e aos preços de mercado, obtendo-se a diferença relativa percentual entre ambos, $F_{(B S) \text { jit }}$ e $\mathrm{F}_{(\mathrm{CEV}) \mathrm{jit}}$. A partir desses resultados, aplicou-se a Equação 11, obtendo-se $\mathrm{R}_{(\mathrm{BS}) \mathrm{t}} \mathrm{e}$ $\mathrm{R}_{(\mathrm{CEV}) t}$ - a média da diferença relativa percentual entre os preços. Os resultados obtidos, para os modelos BS e CEV, respectivamente, podem ser visualizados na Tabela 1 e na Tabela 2 a seguir.

Embora o número de observações, $n$, para cada instante t, seja pequeno em alguns casos (o que prejudica a robustez dos testes), pode-se verificar que, com algumas exceções, a média das diferenças percentuais relativas torna-se menos negativa (ou mais positiva), à medida que $\mathrm{M}$ aumenta, para ambos os modelos: de forma mais generalista, à medida que $\mathrm{M}$ aumenta, o grau de subavaliação diminui. Entretanto os valores obtidos para a média das diferenças percentuais relativas, para o modelo $\mathrm{CEV}$, apresentam-se mais distantes de zero do que no modelo BS. 
Tabela 1: Black e Scholes x Mercado

\begin{tabular}{r|r|r|r|r|r|r|r|r|r}
\hline $\mathrm{t}$ & \multicolumn{3}{|c|}{$\mathrm{M} \leq 0,95$} & \multicolumn{3}{c|}{$0,95<\mathrm{M} \leq 1,05$} & \multicolumn{3}{|c}{$\mathrm{M}>1,05$} \\
\hline & $\mathrm{R}_{(\mathrm{BS}) \mathrm{t}}$ & $\mathrm{t}$ de Student & $\mathrm{n}$ & \multicolumn{1}{c|}{$\mathrm{R}_{(\mathrm{BS}) \mathrm{t}}$} & $\mathrm{t}$ de Student & $\mathrm{n}$ & $\mathrm{R}_{(\mathrm{BS}) \mathrm{t}}$ & $\mathrm{t}$ de Student & $\mathrm{n}$ \\
\hline 60 & - & - & - & - & - & - & $-0,50 \%$ & $(0,48)$ & 7 \\
\hline 55 & $8,53 \%$ & 0,62 & 4 & $4,33 \%$ & 1,16 & 7 & $-1,89 \%$ & $(1,07)$ & 10 \\
\hline 50 & $-6,79 \%$ & $(1,45)$ & 15 & $0,30 \%$ & 0,07 & 14 & $1,15 \%$ & 0,92 & 23 \\
\hline 45 & $-15,61 \%$ & $(3,86)$ & 16 & $-0,73 \%$ & $(0,51)$ & 30 & $2,30 \%$ & 1,89 & 33 \\
\hline 40 & $-6,48 \%$ & $(2,39)$ & 21 & $-0,95 \%$ & $(0,84)$ & 36 & $1,70 \%$ & 2,65 & 30 \\
\hline 35 & $11,62 \%$ & 0,76 & 19 & $1,71 \%$ & 0,43 & 35 & $0,40 \%$ & 0,27 & 44 \\
\hline 30 & $-11,24 \%$ & $(1,64)$ & 15 & $-4,59 \%$ & $(2,04)$ & 32 & $-2,71 \%$ & $(0,66)$ & 48 \\
\hline 25 & $-6,15 \%$ & $(2,10)$ & 16 & $-0,88 \%$ & $(0,53)$ & 33 & $-0,72 \%$ & $(1,14)$ & 47 \\
\hline 20 & $-0,62 \%$ & $(0,13)$ & 10 & $-1,81 \%$ & $(0,48)$ & 31 & $-0,72 \%$ & $(0,46)$ & 37 \\
\hline 15 & $3,64 \%$ & 0,37 & 2 & $-4,39 \%$ & $(2,30)$ & 23 & $-3,40 \%$ & $(2,62)$ & 28 \\
\hline 10 & $124,50 \%$ & 1,30 & 2 & $-3,99 \%$ & $(1,10)$ & 19 & $-0,75 \%$ & $(0,10)$ & 35 \\
\hline 5 & - & - & - & $15,53 \%$ & 0,91 & 9 & $-2,28 \%$ & ${ }^{* *}(3,23)$ & 20 \\
\hline
\end{tabular}

Onde $\mathrm{M} £ 0,95$ representa opções fora do preço, $\mathrm{M}>1,05$ representa opções dentro do preço $\mathrm{e}$ $0,95<\mathrm{M} £ 1,05$ representa opções no preço; $\mathrm{R}_{(\mathrm{BS}) \mathrm{t}}$ é média das diferenças percentuais relativas no instante $t$; $t$ é o número de dias úteis para o vencimento da opção.

* resultado significante ao nível de 5\%.

** resultado significante ao nível de $1 \%$.

\section{Tabela 2: CEV x Mercado}

\begin{tabular}{r|r|r|r|r|r|r|r|r|r}
\hline $\mathrm{t}$ & \multicolumn{3}{|c|}{$\mathrm{M} \leq 0,95$} & \multicolumn{3}{c|}{$0,95<\mathrm{M} \leq 1,05$} & \multicolumn{3}{c}{$\mathrm{M}>1,05$} \\
\hline & $\mathrm{R}_{(\mathrm{CEV}) \mathrm{t}}$ & $\mathrm{t}$ de Student & $\mathrm{n}$ & $\mathrm{R}_{(\mathrm{CEV}) \mathrm{t}}$ & $\mathrm{t}$ de Student & $\mathrm{n}$ & $\mathrm{R}_{(\mathrm{CEV}) \mathrm{t}}$ & $\mathrm{t}$ de Student & $\mathrm{n}$ \\
\hline 60 & & & & & & & $-0,44 \%$ & $(0,44)$ & 7 \\
\hline 55 & $6,82 \%$ & 0,51 & 4 & $3,72 \%$ & 0,98 & 7 & $-1,89 \%$ & $(1,07)$ & 10 \\
\hline 50 & $-10,25 \%$ & $(2,04)$ & 15 & $-0,58 \%$ & $(0,14)$ & 14 & $1,18 \%$ & 0,95 & 23 \\
\hline 45 & $-19,98 \%$ & $(4,36)$ & 16 & $-1,74 \%$ & $(1,17)$ & 30 & $2,29 \%$ & 1,89 & 33 \\
\hline 40 & $-9,28 \%$ & $(3,12)$ & 21 & $-1,62 \%$ & $(1,42)$ & 36 & $1,77 \%$ & ${ }^{*} 2,78$ & 30 \\
\hline 35 & $8,59 \%$ & 0,57 & 19 & $1,26 \%$ & 0,31 & 35 & $0,55 \%$ & 0,36 & 44 \\
\hline 30 & $-16,39 \%$ & $(2,23)$ & 15 & $-5,41 \%$ & $(2,54)$ & 32 & $-2,64 \%$ & $(0,52)$ & 48 \\
\hline 25 & $-10,97 \%$ & $(2,47)$ & 16 & $-1,51 \%$ & $(0,89)$ & 33 & $-0,57 \%$ & $(0,89)$ & 47 \\
\hline 20 & $-3,06 \%$ & $(0,68)$ & 10 & $-2,27 \%$ & $(0,60)$ & 31 & $-1,00 \%$ & $(0,61)$ & 37 \\
\hline 15 & $2,09 \%$ & 0,22 & 2 & $-4,70 \%$ & $(2,43)$ & 23 & $-3,32 \%$ & ${ }^{*}(2,55)$ & 28 \\
\hline 10 & $112,81 \%$ & 1,30 & 2 & $-4,43 \%$ & $(1,20)$ & 19 & $-0,65 \%$ & $(0,09)$ & 35 \\
\hline 5 & & & & $14,92 \%$ & 0,82 & 9 & $-2,56 \%$ & ${ }^{* *}(3,65)$ & 20 \\
\hline
\end{tabular}

Onde $\mathrm{M} \leq 0,95$ representa opções fora do preço, $\mathrm{M}>1,05$ representa opções dentro do preço $\mathrm{e}$ $0,95<\mathrm{M} \leq 1,05$ representa opções no preço; $\mathrm{R}_{(\mathrm{CEV}) t}$ é média das diferenças percentuais relativas no instante $\mathrm{t}$; $\mathrm{t}$ é o número de dias úteis para o vencimento da opção.

* resultado significante ao nível de 5\%.

** resultado significante ao nível de $1 \%$. 
Para opções mais próximas do vencimento $(\mathrm{t} \leq 30)$, os modelos subavaliaram praticamente todas as opções. Para opções mais distantes do vencimento $(t \geq$ 35), os modelos subavaliaram as opções fora do preço e no preço, superavaliando as opções que se encontravam dentro do preço.

Por fim, dos 33 valores obtidos para $\mathrm{R}_{(\mathrm{BS}) \mathrm{t}}$, verifica-se, pelo teste $\mathrm{t}$, que 7 apresentaram resultados estatisticamente diferentes de zero; desses, 2 foram significativamente diferentes ao nível de $1 \%$ e 5 ao nível de 5\%. Para as 33 observações de $\mathrm{R}_{(\mathrm{CEV}) t}, 9$ são significativamente diferentes de zero, sendo 3 significativamente diferentes ao nível de significância de 1\%.

Embora os resultados sugiram que os modelos se ajustem melhor às opções que se encontram no preço, uma possível explicação estaria baseada na forma como foi realizada a estimativa dos parâmetros $\sigma, \theta$ e $\delta$. Como eles são estimados para opções no preço, pode-se imaginar que os preços teóricos, calculados a partir dessas estimativas, se ajustem mais adequadamente aos preços de mercado para opções no preço.

Percebe-se, em ambos os modelos, que, quanto mais fora do preço, maior a subavaliação. Ressalta-se que, por estarem sendo utilizadas diferenças percentuais, os desvios, para opções fora do preço, apresentam-se maiores, porquanto, nesta faixa se encontram as opções com menores preços. Como os preços observados no mercado não são contínuos (os preços ficam limitados aos centésimos), a variação percentual, nestes casos, tende a ser maior, apesar de terem sido eliminadas as opções com preços inferiores a $\mathrm{R} \$ 1,00$.

Por fim, o resultado do teste comparativo direto, entre os modelos de Black e Scholes (1973) e da CEV, encontra-se na Tabela 3, apresentada na seqüência. Das 33 observações para $\mathrm{R}_{\mathrm{mt}}, 18$ apresentam resultados significativamente diferentes de zero, sendo 16 deles diferentes de zero ao nível de significância de $1 \%$.

Verifica-se que o modelo CEV, em relação ao modelo BS, subavalia as opções que se encontram fora do preço e no preço e superavalia as opções dentro do preço. Pode-se observar, na Figura 1 a seguir, o gráfico de dispersão para os valores de $\mathrm{F}_{\text {mijt }}$ e de $\mathrm{M}$, confirmando os resultados obtidos.

Com base nos testes realizados nesta seção, pode-se concluir que, para a amostra estudada, o modelo CEV subavalia as opções de compra no preço e fora do preço de forma mais intensa que o modelo BS. O inverso é verdadeiro para as opções dentro do preço; ainda assim, comparando-se a Tabela 1 e a Tabela 2, verifica-se que o desempenho de ambos os modelos, relativamente ao mercado, foi semelhante, para $\mathrm{M}>1,05$. 
Tabela 3: Black e Scholes x CEV

\begin{tabular}{r|r|r|r|r|r|r|r|r|c}
\hline $\mathrm{t}$ & \multicolumn{3}{|c|}{$\mathrm{M} \leq 0,95$} & \multicolumn{3}{c|}{$0,95<\mathrm{M} \leq 1,05$} & \multicolumn{3}{c}{$\mathrm{M}>1,5$} \\
\hline & $\mathrm{R}_{\mathrm{mt}}$ & $\mathrm{t}$ de Student & $\mathrm{n}$ & $\mathrm{R}_{\mathrm{mt}}$ & $\mathrm{t}$ de Student & $\mathrm{n}$ & $\mathrm{R}_{\mathrm{mt}}$ & $\mathrm{t}$ de Student & $\mathrm{n}$ \\
\hline 60 & & & & & & & $0,07 \%$ & 0,95 & 7 \\
\hline 55 & $-1,58 \%$ & ${ }^{* *}(7,84)$ & 4 & $-0,60 \%$ & ${ }^{* *}(4,21)$ & 7 & $0,01 \%$ & 0,26 & 10 \\
\hline 50 & $-3,93 \%$ & $(2,21)$ & 15 & $-1,07 \%$ & $(1,92)$ & 14 & $0,04 \%$ & 1,26 & 23 \\
\hline 45 & $-5,85 \%$ & $(3,64)$ & 16 & $-1,03 \%$ & $(4,45)$ & 30 & $0,00 \%$ & $(0,03)$ & 33 \\
\hline 40 & $-3,22 \%$ & $(3,20)$ & 21 & $-0,67 \%$ & ${ }^{* *}(4,12)$ & 36 & $0,07 \%$ & ${ }^{* *} 2,81$ & 30 \\
\hline 35 & $-2,95 \%$ & $(7,06)$ & 19 & $-0,46 \%$ & ${ }^{* *}(5,47)$ & 35 & $0,14 \%$ & ${ }^{* *} 7,72$ & 44 \\
\hline 30 & $-7,90 \%$ & $(2,40)$ & 15 & $-0,79 \%$ & $(2,71)$ & 32 & $0,07 \%$ & 0,01 & 48 \\
\hline 25 & $-5,50 \%$ & $(1,68)$ & 16 & $-0,66 \%$ & ${ }^{* *}(3,42)$ & 33 & $0,14 \%$ & ${ }^{* *} 4,50$ & 47 \\
\hline 20 & $-2,41 \%$ & $(7,94)$ & 10 & $-0,55 \%$ & $(3,67)$ & 31 & $-0,29 \%$ & $(0,76)$ & 37 \\
\hline 15 & $-1,45 \%$ & $(3,69)$ & 2 & $-0,35 \%$ & ${ }^{* *}(3,02)$ & 23 & $0,08 \%$ & ${ }^{* *} 5,63$ & 28 \\
\hline 10 & $-4,16 \%$ & $(1,69)$ & 2 & $-0,53 \%$ & $(1,79)$ & 19 & $0,09 \%$ & 0,02 & 35 \\
\hline 5 & & & & $-0,77 \%$ & $(1,16)$ & 9 & $-0,28 \%$ & $(1,69)$ & 20 \\
\hline
\end{tabular}

Onde $\mathrm{M} \leq 0,95$ representa opções fora do preço, $\mathrm{M}>1,05$ representa opções dentro do preço $\mathrm{e}$ $0,95<\mathrm{M} \leq 1,05$ representa opções no preço; $\mathrm{R}_{\mathrm{mt}}$ é média das diferenças percentuais relativas dos modelos no instante $\mathrm{t}$; $\mathrm{t}$ é o número de dias úteis para o vencimento da opção.

* resultado significante ao nível de 5\%.

** resultado significante ao nível de $1 \%$.

\section{Figura 1: Fm x M}

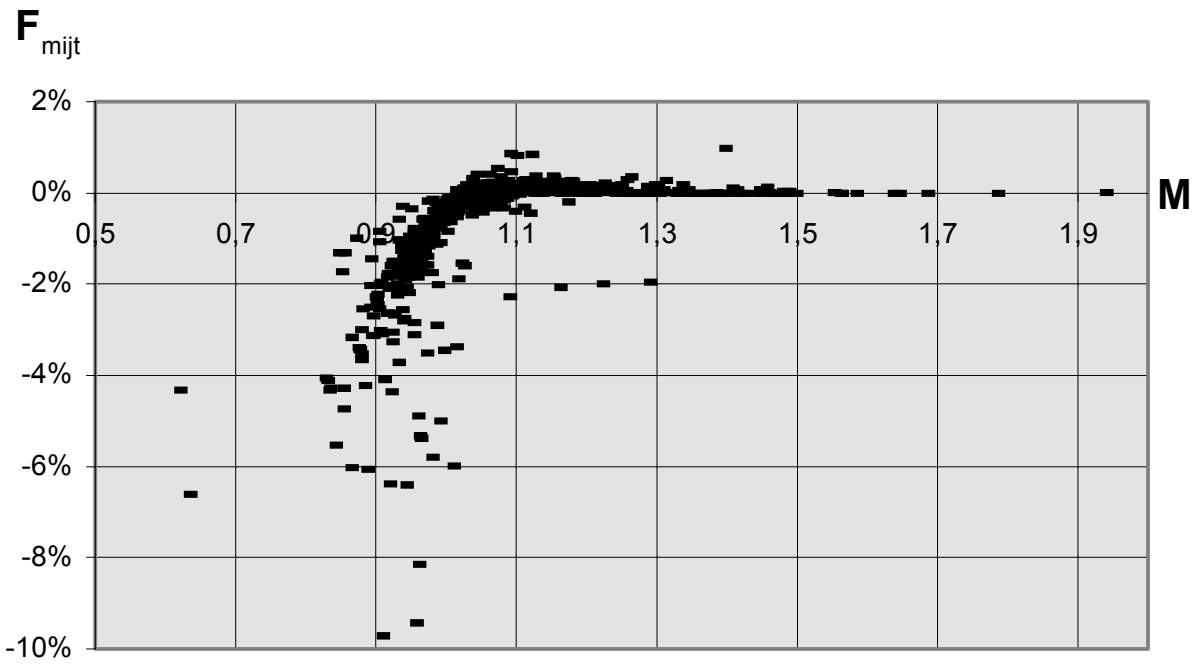




\section{CONCLUSÃo}

Com base nos resultados, pode-se afirmar que o modelo BS se ajustou melhor do que o modelo CEV na precificação das opções de compra negociadas no mercado brasileiro, no período de julho de 1994 a junho de 1997.

Os resultados da aplicação do modelo BS apresentam-se, de maneira geral, em conformidade com os obtidos por Black (1975) e Barreto e Baidya (1987), por exemplo, e opostos aos obtidos por Macbeth e Merville (1979). Os resultados do modelo CEV, por sua vez, foram opostos aos obtidos por Macbeth e Merville (1980), Peterson e Scott (1988) e Hauser e Lauterbach (1997). Na realidade, o modelo CEV aumentou os vieses observados no modelo BS, para opções fora do preço e no preço, embora tenha reduzido, ainda que tenuamente, o viés para os casos em que $\mathrm{M}>1,05$.

De forma mais ampla, os resultados sugerem que, para a amostra utilizada, o processo de difusão log-normal explica melhor o comportamento das ações do que o processo de difusão com a elasticidade da variância constante.

Em parte, os resultados obtidos justificam-se pelo fato de que os preços das opções negociadas no mercado são determinados, em última instância, pelos agentes econômicos, que compram e vendem ações e opções. Logo, pode-se supor que, se esses agentes, na valoração das opções de compra, se utilizam do modelo BS, seria de se esperar que, em um teste como o realizado, tal modelo se ajustasse melhor do que qualquer outro modelo.

Pode-se dizer, portanto, que o esforço computacional extra, na estimativa dos parâmetros relevantes do modelo $\mathrm{CEV}$, não é justificável, visto que o modelo BS, além de ser menos complexo, ajustou-se melhor aos preços das opções da amostra.

\section{Notas}

${ }^{1}$ Ativo derivativo é aquele cujo valor é função do valor de outra(s) variável(is).

${ }^{2}$ De acordo com Bergman, Grundy e Wiener (1996, p. 1574) “uma classe de processos estocásticos, que tradicionalmente tem desempenhado papel importante na modelagem da dinâmica dos preços dos ativos objetos, é a dos processos de difusão". Isaacson e Madsen (1976) definem processo estocástico como sendo uma família de variáveis randômicas definidas sobre o mesmo espaço amostral. Para Karlin e Taylor (1981, p. 157), "processo estocástico com parâmetro em tempo contínuo, que possui a (forte) propriedade de Markov e para a qual os caminhos amostrais, $\mathrm{X}_{\mathrm{t}}$, são (quase sempre) funções contínuas de t, é chamado processo de difusão". 
${ }^{3}$ Uma opção de compra encontra-se: dentro do preço, quando o preço do ativo-objeto estiver acima do preço de exercício; fora do preço, quando o preço do ativo-objeto estiver abaixo do preço de exercício; no preço, quando o preço do ativo-objeto for igual ao preço de exercício.

${ }^{4}$ Variância implícita é definida por Manaster e Koehler (1982, p. 227) como “o valor da variância instantânea dos retornos da ação que, quando aplicado na fórmula de Black-Scholes, resulta em um preço de modelo igual ao preço de mercado".

${ }^{5}$ Pelo fato de os preços observados no mercado não serem contínuos, a limitação em duas casas após a vírgula prejudica a comparação percentual. Procurando contornar este problema, seguindo os trabalhos de Lauterbach e Schultz (1990) e Hauser e Lauterbach (1997), optou-se por eliminar as opções com preços inferiores a $\mathrm{R} \$ 1,00$.

${ }^{6}$ Após a aplicação da Equação 8, foram observadas 16 estimativas para o parâmetro $\theta$ que violaram a restrição $0 \leq \theta<2$, e que, portanto, foram excluídas da amostra.

\section{ReferênCIAS Bibliográficas}

BARRETO, L. A. DE B.;

BAIDYA, T. K. N.

Teste empírico do modelo de Black e Scholes na avaliação de opções da Vale do Rio Doce. Revista Brasileira de Mercado de Capitais, v. 13, n. 39, p. 89-108, abr./jun. 1987.

BECKER, J. L.;

LEMGRUBER, E. F.

A trading strategy analysis of the Brazilian option market : evidence from the three most traded call options. COPPEAD, 1987.

BERGMAN, Y;

GRUNDY, B.;

WIENER, Z.

General properties of option prices.

The Journal of Finance, v. 51, n. 5, p.1573-1610, Dec. 1996.
BLACK, F.

Fact and fantasy in the use of options. Financial Analysts Journal, p. 36-72, July/Aug. 1975.

BLACK, F.;

SCHOLES, M.

The valuation of option contracts and a test of market efficiency. The Journal of Finance, p. 399417, May 1972.

The pricing of options and corporate liabilities. Journal of Political Economy, p.637-654, May/June 1973.

COX, J.

The constant elasticity of variance pricing model. The Journal of Portfolio Managment, p. 15-17, 1996. 
COX, J.;

ROSS, S. A.

The valuation of options for alternative stochastic processes. Journal of Financial Economics, n. 3, p.145-166, Jan./Mar. 1976.

FORTUNA, E.

Mercado financeiro : produtos e serviços. Rio de Janeiro : Qualitymark, 1996.

HAUSER, S.;

LAUTERBACH, B.

The relative performance of five alternative warrant pricing models. Financial Analysts Journal, p. 5561, Jan./Feb. 1997.

\section{HULL, J.}

Options, futures, and other derivative securities. 2 . ed. Englewood Cliffs, NJ : PrenticeHall, 1993.

ISAACSON, D. L.;

MADSEN, R. W.

Markov chains : theory and applications. New York : John Wiley \& Sons, 1976.

KARLIN, S.;

TAYLOR, H. M.

A second course in stochastic processes. London : Academic Press, 1981.

LAUTERBACH, B.; SCHULTZ, P.

Pricing warrants : an empirical study of the Black-Scholes model and its alternatives. The Journal of Finance, v. 45, n. 4, p.11811209, Sept. 1990.

MACBETH, J. D.;

MERVILLE, L. J.

An empirical examination of the Black-Scholes call option pricing model. The Journal of Finance, v. 34, n. 5, p. 1173-1186, Dec. 1979.

Tests of the Black-Scholes and Cox call option valuation models. The Journal of Finance, v. 35, n. 2, p. 285-301, May 1980.

MANASTER, S.;

KOEHLER, G.

The calculation of implied variances from the Black-Scholes model : a note. The Journal of Finance, v. 37, n. 1, p.227-230, Mar. 1982.

MERTON, R. C.

Theory of rational option pricing. Bell Journal of Economics and Management Science, v. 4, p. 141-183, Spring 1973.

SWIDLER, S.;

DILTZ, D.

Implied volatilities and transaction costs. Journal of Financial and Quantitative Analysis, v. 27, n. 3, p. 437-447, Sept. 1992.

SCHOLES, M.

Taxes and the pricing of options. The Journal of Finance, v. 31, n. 2, p. 319-332, May 1976. 
TUCKER, A.;

PETERSON, D.;

SCOTT, E.

Tests of the Black-Scholes and constant elasticity of variance currency call option valuation models. The Journal of Financial Research, v. 11, n. 3, p. 201-213, Autumm 1988. 